\title{
Realização do timeout pela equipe cirúrgica: facilidades e dificuldades
}

\author{
Surgical timeout protocol: facilities and difficulties \\ Realización del timeout del equipo quirúrgico: facilidades y dificultades
}

Glaucia Stein Martins ${ }^{1}$, Rachel de Carvalho ${ }^{2}$

RESUMO: Objetivo: verificar a opinião da equipe cirúrgica sobre a realização do timeout e identificar as facilidades e dificuldades para sua aplicação. Método: estudo exploratório, descritivo com abordagem quantitativa realizado em um Centro Cirúrgico de uma instituição privada de São Paulo, com 39 profissionais, por meio da aplicação de um questionário estruturado. Resultados: todos os participantes (100\%) conhecem o timeout e a maioria (92,3\%) reconhece sua eficácia, acredita que a aplicação traz segurança para o cliente (94,9\%) e para a própria equipe. Os fatores que facilitam a aplicação do timeout são: colaboração da equipe, conhecimento, clareza e objetividade do protocolo; os que dificultam são a falta de colaboração da equipe, protocolo longo e repetitivo, e atrasos dos médicos e das cirurgias. Conclusão: a implementação do timeout é um desafio para a instituição e para a equipe, porém o protocolo contribui para redução de riscos e de eventos adversos, proporcionando segurança ao cliente.

PALAVRAS-CHAVE: Segurança do paciente. Cirurgia. Procedimentos cirúrgicos operatórios. Equipe de enfermagem. Equipe de assistência ao paciente.

ABSTRACT: Objective: this study aimed to verify the opinion of the surgical team about the completion of timeout and identify the advantages and difficulties of its application. Method: it is an explanatory, descriptive study of quantitative approach carried out through the application of a structured questionnaire to 39 health professionals at the Surgical Center of a private institution in Sao Paulo. Results: all respondents $(100 \%)$ know what timeout is; and most of them acknowledge its effectiveness $(92,3 \%)$ and believe that its application provides safety to patients and the team itself $(94,9 \%)$. The factors that facilitate the implementation of surgical timeout are collaboration of medical staff and knowledge, clarity and objectivity of the protocol; and those that hamper its implementation are lack of team collaboration, duration and repetitiveness, and delays of physicians and surgeries. Conclusion: implementation of timeout protocol is challenge for institutions and surgical teams, but contributes to reduced risks and adverse events, providing safety to the client.

KEYWORDS: Patient/surgery safety. Surgical procedures. Nursing staff. Patient care team.

RESUMEN: Objetivo: verificar la opinión del equipo quirúrgico sobre la realización del timeout e identificar las facilidades y dificultades para su aplicación. Método: estudio exploratorio, descriptivo, con enfoque cuantitativo, realizado en un Centro Quirúrgico de una institución privada de São Paulo, con 39 profesionales, mediante la aplicación de un cuestionario estructurado. Resultados: todos los participantes (100\%) conocen el timeout y la mayoría (92,3\%) reconoce su eficacia, cree que la aplicación aporta seguridad al cliente $(94,9 \%)$ y al propio equipo. Los factores que facilitan la aplicación del timeout son: la colaboración del equipo, el conocimiento, la claridad y objetividad del protocolo; mientras que aquellos que la dificultan son: la falta de colaboración del equipo, el protocolo largo y repetitivo, y los retrasos tanto de los médicos como de las cirugías. Conclusión: la implementación del timeout es un desafío para la institución y para el equipo; sin embargo, el protocolo contribuye a la reducción de riesgos y de eventos adversos, proporcionando, así, mayor seguridad al cliente.

PALABRAS ClAVE: Seguridad del paciente. Cirugía. Procedimientos quirúrgicos operativos. Equipo de enfermería. Equipo de atención al paciente.

${ }^{1}$ Enfermeira. Graduada pela Faculdade de Enfermagem do Hospital Israelita Albert Einstein (FEHIAE). E-mail: glaucia.stein@yahoo.com.br

${ }^{2}$ Enfermeira. Especialista em Cardiologia e Centro Cirúrgico. Mestre e Doutora em Enfermagem. Escola de Enfermagem da USP.

Docente dos Cursos de Graduação e Pós-Graduação da FEHIAE. Coordenadora do Curso de Pós-Graduação da FEHIAE.

Av. Prof Francisco Morato, 4293, Butantã, CEP 05521-000, São Paulo, SP, Brasil.

Telefone: (11) 9545-9948. E-mail: rachel.carvalho@einstein.br 


\section{Introdução}

Há alguns séculos, a cirurgia tem se destacado pelo mundo como componente essencial ao cuidado da saúde, indicando que a segurança é primordial ${ }^{1}$. Em 1999, o Institute of Medicine (IOM) publicou um relatório que alertava a comunidade científica sobre o alto índice do impacto das perdas humanas nos Estados Unidos, decorrentes de erros que poderiam ser evitados antes da cirurgia. A partir de então, houve progresso significativo nos estudos relacionados ao tema ${ }^{2}$.

Devido às evidências de erros em escala mundial, a 55. ${ }^{a}$ Assembleia da Saúde de 2002 adotou a Resolução 'Qualidade do cuidado: segurança do paciente' (World Health Assembly - Quality of care: patient safety - WHA55.18) ${ }^{3}$, que estimula os países a fortalecer a segurança da assistência à saúde. Em maio de 2004, a 57. ${ }^{a}$ Assembleia criou uma aliança internacional, formada pela Organização Mundial de Saúde (OMS) e pela Organização Pan-Americana de Saúde (OPAS). Suas ações foram concentradas em campanhas de segurança do paciente, identificando soluções e desenvolvendo iniciativas de relatos de aprendizagem, para poder salvar milhões de vidas ${ }^{1}$.

Em 2010, divulgou-se que houve uma queda na taxa de mortalidade decorrente de erros em cirurgias e que as complicações diminuíram de $35,2 \%$ para $24,3 \%$. O cheklist proposto pela aliança internacional não só impactou no resultado como também melhorou a comunicação entre a equipe cirúrgica ${ }^{4}$.

A OMS criou o manual 'Cirurgias Seguras Salvam Vidas', que reforça práticas de segurança em todas as etapas do perioperatório e que potencializa a probabilidade de melhoria nos resultados. Este manual foi guiado por princípios simples e de fácil compreensão, de ampla aplicabilidade, podendo ser usado em qualquer lugar do mundo. Tem como meta melhorar a assistência cirúrgica, uma vez que checa a segurança do cliente em todo o período perioperatório ${ }^{1}$.

O 'Protocolo Universal para Prevenção de Cirurgias com Local de Intervenção Errado, Procedimento Errado ou Pessoa Errada' da Joint Commission International (JCI) preconiza a marcação do local da intervenção cirúrgica, o processo de verificação pré-operatório e uma pausa realizada imediatamente antes do início do procedimento, denominada timeout. Foi aprovado por mais de 50 associações e organizações profissionais e, em 2010, foi revisto, permitindo melhor flexibilidade na sua aplicação $0^{5,6}$.

O timeout faz parte desse programa e da $4 .^{a}$ Meta Internacional de Segurança do Paciente da JCI. Seu objetivo é assegurar que seja realizado o procedimento correto, no paciente correto e no local correto, de modo a proporcionar um cuidado seguro e de alta qualidade, permitindo que todas as questões não respondidas ou confusas sejam resolvidas. É uma prática baseada em evidências e opiniões de especialistas, que vem ajudar a garantir que a equipe cirúrgica possa seguir alguns passos de segurança e, assim, minimizar os riscos mais comuns evitáveis para o paciente ${ }^{5-7}$.

A cultura de segurança do paciente, conforme proposto pela OMS, é definida por três fases: check in, timeout e check out ${ }^{8}$.

O timeout, objeto deste estudo, deve ser realizado já com o paciente em sala operatória (SO), imediatamente antes da incisão na pele. Neste momento, o Enfermeiro ou o circulante de sala confere, em voz alta, na presença do Médico Cirurgião, do Médico Auxiliar, do Anestesiologista, do Instrumentador Cirúrgico e do Técnico de Enfermagem, os seguintes itens ${ }^{6-8}$ :

- Antes da indução anestésica, a enfermagem e o Médico Anestesiologista verificam: dois identificadores do paciente; procedimento conforme agendado; sítio cirúrgico demarcado; dificuldades de ventilação ou risco para aspiração; materiais de vias aéreas presentes na sala e em condições de uso; presença ou ausência de alergias; recursos para prevenção de trombose utilizados; risco de perda sanguínea prevista e reserva de sangue; paciente com acesso venoso presente e permeável.

- Antes da incisão cirúrgica, a enfermagem e o Médico Cirurgião apresentam a equipe; verificam membros da equipe presentes; checam dois identificadores do paciente; verificam se o procedimento está conforme; checam se o sítio cirúrgico está demarcado; observam se o antibiótico profilático foi realizado; verificam se os exames estão no prontuário e em sala.

Caso algum item não esteja correto, o procedimento deverá ser interrompido até a regularização do mesmo ${ }^{7-8}$.

Embora a preocupação referente à segurança do paciente cirúrgico ser difundida por organizações governamentais s,-4, $^{1,9}$ e por instituições de saúde particulares ${ }^{7,8}$, percebe-se um número crescente de publicações de estudiosos acerca do assunto $^{2,10,11}$, porém ainda incipientes no Brasil.

Considerando-se a importância dos conceitos aqui mencionados e a maior aplicabilidade dos protocolos de checagem relacionados aos mais diversos procedimentos anestésico-cirúrgicos, esta pesquisa tem como finalidade discutir aspectos relacionados à realização do timeout na SO pela equipe cirúrgica, de modo que possa contribuir com informações que orientem os profissionais de saúde e assegurem segurança aos pacientes cirúrgicos.

\section{Objetivos}

- Verificar a opinião da equipe cirúrgica sobre a realização do timeout em sala operatória, para o paciente e para o profissional;

- Identificar as facilidades e as dificuldades na realização do timeout pela equipe cirúrgica, imediatamente antes do início do procedimento cirúrgico. 


\section{Método}

Esta pesquisa se caracteriza como um estudo de campo, descritivo-exploratório, com abordagem quantitativa . Foi desenvolvido no Centro Cirúrgico (CC) de uma instituição privada de São Paulo, que conta com 14 salas operatórias, em que são realizadas cerca de duas mil cirurgias por mês. No CC, sede do estudo, o timeout foi implantado no segundo semestre de 2010, sendo aplicado em todos os procedimentos.

A amostra foi composta por 39 integrantes da equipe cirúrgica, sendo: 14 Médicos, 14 Técnicos de Enfermagem, 07 Enfermeiros e 04 Instrumentadores Cirúrgicos. Os critérios de inclusão dos sujeitos na amostra foram: pertencer à equipe cirúrgica (Cirurgião, Anestesiologista, Instrumentador Cirúrgico, Enfermeiro e Técnico de Enfermagem); atuar no CC e participar da realização do timeout; estar presente na sala de operações, no momento da realização do timeout, imediatamente antes do início do procedimento cirúrgico; consentir fazer parte da pesquisa, por meio de leitura e assinatura do Termo de Consentimento Livre e Esclarecido - TCLE.

$\mathrm{O}$ instrumento de coleta de dados foi composto por um questionário estruturado preenchido pelos membros da equipe; o questionário apresenta duas partes: 1) caracterização biossocial da amostra e 2) levantamento da opinião acerca da realização do timeout pela equipe, no qual também foram identificadas as facilidades e as dificuldades da sua aplicação.

A coleta de dados foi realizada por meio de abordagem direta a cada um dos membros da equipe, após aprovação do projeto pela Comissão Científica e pelo Comitê de Ética em Pesquisa da Instituição, sob protocolo Plataforma Brasil número CAAE - 03505012.8.0000.0071, bem como após autorização da gestora do CC.

\section{Resultados}

A amostra do estudo foi composta por 39 profissionais pertencentes à equipe cirúrgica, sendo 14 Médicos Cirurgiões e Anestesiologistas (35,9\%); 14 Técnicos de Enfermagem
$(35,9 \%)$, que atuam como circulantes de sala operatória; 07 Enfermeiros $(17,9 \%)$ e 04 Instrumentadores Cirúrgicos $(10,3 \%)$.

Quanto ao gênero, 23 profissionais $(59,0 \%)$ são do sexo feminino e $16(41,0 \%)$ do sexo masculino. O tempo de profissão e o tempo de atuação em CC variaram de menos de um ano a mais de 30 anos, havendo predominância da faixa dos 06 aos 10 anos (11 ou 28,2\%), tanto para formação quanto para atuação em CC.

Foi possível identificar a opinião de cada profissional acerca do timeout e para uma fácil e melhor visualização dos resultados, trabalhamos os dados de acordo com cada uma das questões do questionário.

Para a Questão 1, no questionamento sobre o conhecimento do timeout, obtiveram-se $100 \%$ de respostas positivas, ou seja, todos os 39 participantes da pesquisa responderam sim, mostrando que a rotina de aplicação do protocolo faz parte do cotidiano destes profissionais.

Quanto à eficácia da utilização do timeout (Questão 2), a grande maioria dos profissionais (36 ou 92,3\%) acredita que o instrumento seja eficaz, porém 03 deles $(7,7 \%)$ responderam que acreditam em parte na eficácia do timeout.

Sobre o timeout trazer segurança ao cliente cirúrgico (Questão 3), 37 participantes $(94,9 \%)$ responderam que sim, concordando com esta afirmação, enquanto $02(5,1 \%)$ responderam concordar em parte, sendo estes últimos médicos.

Já com relação ao timeout trazer segurança à equipe cirúrgica (Questão 4), 35 profissionais $(89,7 \%$ ) acreditam que sim e $04(10,3 \%)$ concordam em parte com essa proposição, sendo estes 02 médicos e 02 técnicos de enfermagem.

Para a Questão 5, que diz respeito à frequência com que o timeout é aplicado, 33 respondentes $(84,6 \%)$ referiram que sempre aplicam o timeout e $06(15,4 \%)$ aplicam frequentemente (Figura 1).

Sobre a complexidade do timeout e a facilidade de sua compreensão (Questão 6), 27 integrantes da equipe (69,2\%) responderam que sim, consideram o timeout simples e de fácil compreensão. Porém, 07 (17,9\%) acreditam em parte

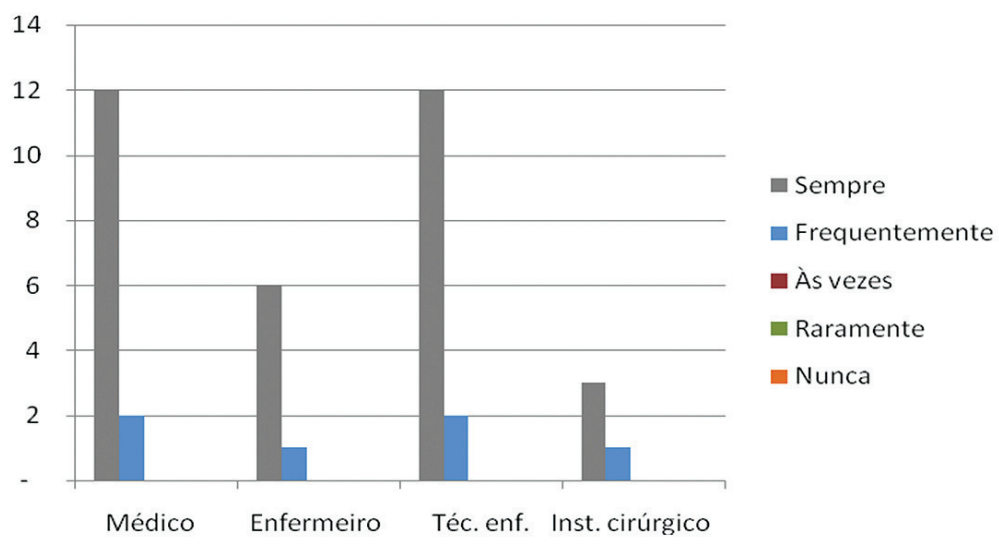

Figura 1. Frequência de aplicação do timeout pela equipe cirúrgica. 
e $05(12,8 \%)$ responderam que o timeout não é simples e nem de fácil compreensão (Figura 2). Todos os profissionais que responderam não para essa pergunta eram Técnicos de Enfermagem.

Com relação ao timeout poder ser utilizado em qualquer procedimento anestésico-cirúrgico (Questão 7), 25 participantes $(64,1 \%)$ responderam concordar, enquanto 13 $(33,3 \%)$ concordam em parte. A maior parte dos Enfermeiros (04) concorda em parte com a aplicação do timeout antes de qualquer procedimento ou situação. Um profissional $(2,6 \%)$ não respondeu a esta questão.

Ao questionar se o timeout facilita a comunicação entre os membros da equipe (Questão 8), a maioria dos participantes (31 ou 79,5\%) respondeu que sim, facilita; 05 $(12,8 \%)$ consideram que o instrumento facilita em parte a comunicação e $03(7,7 \%)$ acham que o timeout não facilita a comunicação entre a equipe (Figura 3 ).

As três últimas perguntas do questionário (Questões 9, $10,11)$ exigiam dos participantes uma resposta dissertativa e subjetiva. Essas questões se referiam à opinião sobre três fatores que julgavam facilitar e outros três que dificultavam a aplicação do timeout no $\mathrm{CC}$, e, por último, deveriam descrever as críticas e/ou sugestões sobre o assunto. Nem todos os participantes responderam a essas questões.

Do total de participantes (39 ou 100,0\%), 03 (7,7\%) não responderam duas perguntas integralmente (Questões 9 e 11 ou Questões 10 e 11) e 22 profissionais $(56,4 \%)$ não responderam integralmente a Questão 11, que solicitava críticas e sugestões.

A Questão 9 fazia referência aos fatores facilitadores da aplicação do timeout e a Questão 10, aos fatores que dificultavam a aplicação do protocolo. Ambas foram respondidas integral ou parcialmente por 38 participantes $(97,4 \%)$.

A repetição de opiniões, termos ou frases semelhantes, nas respostas dos profissionais, permitiu a elaboração de quadros que demonstram os fatores que facilitam a aplicação do timeout (Quadro 1) e os que dificultam sua aplicação (Quadro 2). Vale ressaltar que cada participante poderia responder de um a três fatores que acredita facilitar ou dificultar a aplicação do protocolo.

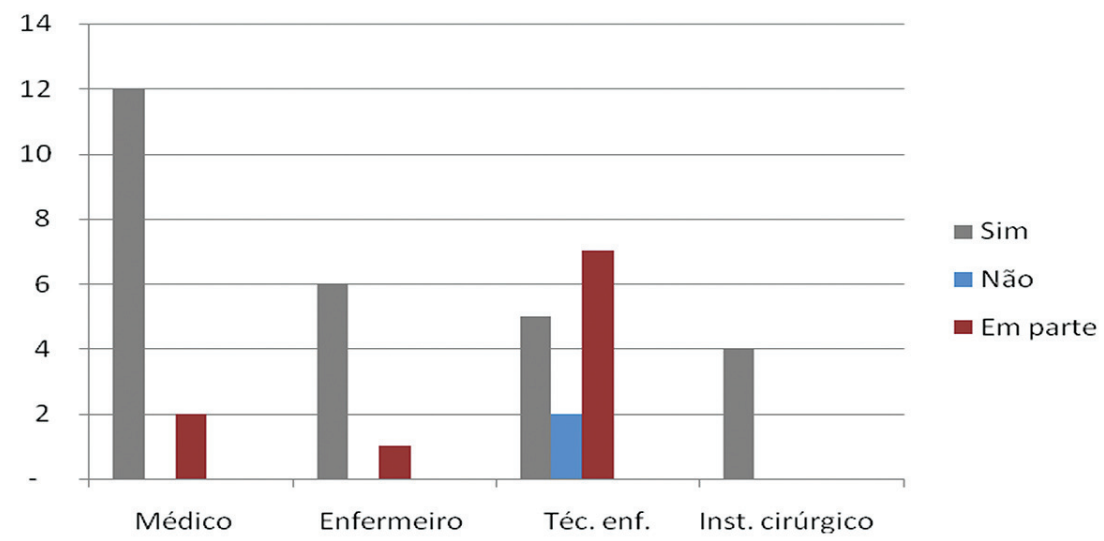

Figura 2. Complexidade e clareza do timeout, segundo a opinião dos membros da equipe cirúrgica.

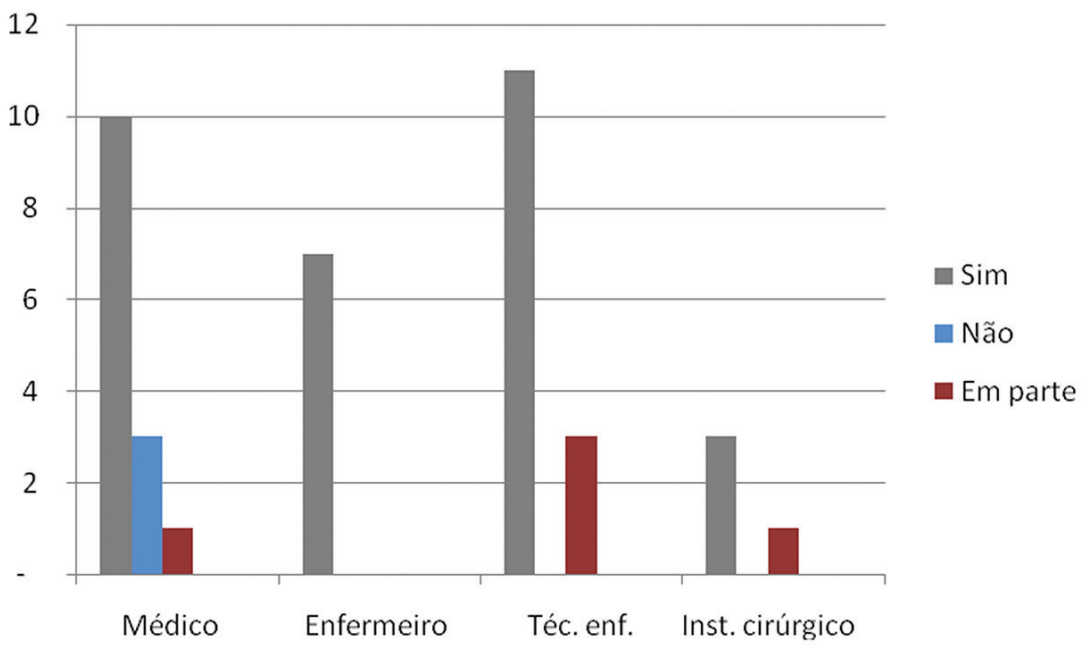

Figura 3. Aplicação do timeout e facilidade de comunicação entre os membros equipe. 
Quadro 1. Fatores que facilitam a aplicação do timeout no Centro Cirúrgico, segundo a opinião dos profissionais, divididos por categorias

\begin{tabular}{|c|c|c|c|c|}
\hline $\begin{array}{l}\text { Categoria profissional / } \\
\text { Fatores que facilitam a aplicação do timeout }\end{array}$ & Médico & Enfermeiro & $\begin{array}{l}\text { Técnico de } \\
\text { Enfermagem }\end{array}$ & $\begin{array}{l}\text { Instrumentador } \\
\text { Cirúrgico }\end{array}$ \\
\hline $\begin{array}{l}\text { Colaboração da equipe médica } \\
\text { (aderência ao protocolo, respeito e atenção da equipe) }\end{array}$ & 09 & 05 & 10 & 01 \\
\hline Conhecimento do protocolo (treinamento) & 06 & 04 & 05 & - \\
\hline Protocolo objetivo (clareza e objetividade do timeout) & 06 & 01 & 02 & 01 \\
\hline $\begin{array}{l}\text { Organização da sala cirúrgica } \\
\text { (montagem correta, materiais e equipamentos disponíveis) }\end{array}$ & - & 02 & 04 & - \\
\hline Boa comunicação & 01 & - & 03 & - \\
\hline Equipe em sala no horário correto & 02 & 01 & - & 01 \\
\hline Total & 24 & 13 & 24 & 03 \\
\hline
\end{tabular}

Quadro 2. Fatores que dificultam a aplicação do timeout no Centro Cirúrgico, segundo a opinião dos profissionais, divididos por categorias

\begin{tabular}{|c|c|c|c|c|}
\hline $\begin{array}{l}\text { Categoria profissional / } \\
\text { Fatores que dificultam a aplicação do timeout }\end{array}$ & Médico & Enfermeiro & $\begin{array}{c}\text { Técnico de } \\
\text { Enfermagem }\end{array}$ & $\begin{array}{l}\text { Instrumentador } \\
\text { Cirúrgico }\end{array}$ \\
\hline $\begin{array}{l}\text { Falta de colaboração da equipe médica } \\
\text { (recusa, falta de compromisso, pressa do médico) }\end{array}$ & 02 & 04 & 12 & 01 \\
\hline Timeout longo / repetitivo & 07 & 02 & 04 & - \\
\hline Atrasos (médicos ou cirurgias) & 05 & 02 & 02 & - \\
\hline Cirurgias de emergência & 03 & 02 & 01 & 01 \\
\hline Cirurgias de pequeno porte / rápidas & - & 02 & 03 & - \\
\hline $\begin{array}{l}\text { Montagem incorreta da sala cirúrgica } \\
\text { (falta de materiais e equipamentos) }\end{array}$ & - & 01 & 03 & - \\
\hline Falta de treinamento & 01 & 01 & - & 01 \\
\hline Total & 18 & 14 & 25 & 03 \\
\hline
\end{tabular}

Com relação às críticas e sugestões (Questão 11), 22 profissionais $(56,4 \%)$ não as apontaram e $17(43,6 \%)$ fizeram tais observações, considerando principalmente que se devem adequar algumas perguntas, pois são realizados os mesmos questionamentos para todas as cirurgias, tornando o instrumento longo, repetitivo e com alguns pontos incompatíveis com o procedimento. Das 21 críticas e sugestões apontadas pelos profissionais, 11 delas eram sobre a repetitividade do timeout.

\section{Discussão}

Os resultados foram baseados na análise das respostas de 39 integrantes da equipe cirúrgica, que atuavam no Centro Cirúrgico (CC) da Instituição sede do estudo. Como o timeout está implantado na Instituição desde 2010, era de se esperar que a totalidade dos profissionais respondesse conhecer o protocolo.

A amostra foi composta por 14 Médicos, entre Cirurgiões e Anestesistas, 14 Técnicos de Enfermagem, 07 Enfermeiros e 04 Instrumentadores, sendo a maioria do gênero feminino, com mais de cinco anos de profissão e de atuação no CC.

Considerando-se toda a equipe, 36 acreditam na eficácia da utilização do timeout. É de extrema importância e necessidade a utilização de um instrumento ou mecanismo eficaz na prevenção de erros ou eventos adversos no hospital, particularmente no CC.

Em 2009, foram publicados os primeiros resultados da implementação do checklist do Programa 'Cirurgias Seguras Salvam Vidas', no New England Journal of Medicine, sendo avaliados, inicialmente, apenas dois parâmetros: grandes complicações e mortalidade. As grandes complicações foram reduzidas de 11 para $7 \%$, significando uma queda de $36 \%$, e a mortalidade caiu de 1 para $0,8 \%$, significando uma queda de $47 \%$, comprovando a efetividade da utilização do timeout para o aumento da segurança assistencial ${ }^{12}$.

Na relação do timeout com a segurança do cliente cirúrgico, 37 profissionais concordam que a aplicação do protocolo traz segurança ao paciente. A presença dos incidentes e eventos adversos que comprometem a segurança do paciente constitui um grande desafio para o aprimoramento da qualidade no 
setor saúde. Com vistas à melhoria contínua da assistência hospitalar, deve-se garantir a existência de mecanismos para prevenção e minimização de $\operatorname{erros}^{13}$. Observa-se que a aplicação de instrumentos como este pode minimizar os riscos aos quais o paciente está exposto.

Pode-se afirmar, portanto, que a implementação do timeout não previne somente a realização de cirurgias com local errado, procedimento errado ou pessoa errada, mas procura identificar e corrigir riscos antes do início do procedimento, por meio da identificação de quase erro (near miss), aumentando a barreira de segurança e a qualidade da assistência. Os gestores e toda a equipe devem assumir atitudes que valorizem a busca de falhas, de maneira preventiva, e não somente a obtenção da falha no resultado final. Nesse contexto, o paciente tornou-se mais contestador e exigente, forçando uma mudança de atitude dos prestadores de serviços ${ }^{11,14}$.

Com relação à segurança da equipe cirúrgica, 35 profissionais acreditam que o timeout pode trazer segurança para eles próprios. Um estudo realizado pelo Institute of Medicine Report To Err is Human: Building a Safer Health System relata que o erro originado por fator humano é o mais comum e este interage com o meio ambiente. O desempenho da equipe é influenciado por emoções, ambiente da sala operatória, estresse, interação entre os membros da equipe e interpretação de comunicação. Desta forma, a melhoria das características da equipe ajuda no processo de comunicação e reduz os danos ao paciente. Dados substanciais sugerem que pelo menos metade de todas as complicações cirúrgicas é evitável e atribuída a erros humanos, podendo causar incapacidade, necessidade de intervenção cirúrgica ou óbito ${ }^{1,15,16}$

Com o surgimento de estudos epidemiológicos acerca de eventos adversos, a conscientização sobre a segurança dos pacientes aumentou nos últimos anos e numerosos esforços têm sido realizados para melhorar a segurança. Muitos desses esforços coincidiram com iniciativas bem sucedidas em hospitais comprometidos ${ }^{16}$.

Assim sendo, organizações internacionais ${ }^{1,3-6}$ recomendam a adoção do checklist, que contém informações sobre o local cirúrgico correto, o procedimento correto e o paciente correto, visando cumprir critérios básicos para a qualidade da assistência, de modo a reduzir as complicações e a mortalidade. Certamente, essa medida traz segurança não só para o paciente, mas também para a equipe cirúrgica, aumentando a confiabilidade e proporcionando subsídios para que se possam instituir estratégias que resultem em melhoria contínua em todas as etapas do procedimento cirúrgico ${ }^{11,17}$.

No CC em que foi realizada a pesquisa, a aplicação do timeout faz parte de um protocolo e foi instituído como rotina desde 2010, devendo, portanto, ser aplicado a todos os clientes. Quanto a este aspecto, obtive-se um resultado de 33 integrantes da equipe que afirmaram sempre aplicar o timeout e 06 integrantes o aplicam frequentemente.
Entre os pesquisados, a maioria (27) considera o timeout simples e de fácil compreensão, porém 07 consideram em parte e 05 não acham o timeout simples e nem fácil. Todos os profissionais que responderam não acharem o timeout simples nem de fácil compreensão são Técnicos de Enfermagem. Desses dados, pode-se inferir que se faz necessária uma intervenção no sentido de sanar as dúvidas e esclarecer todos os itens do timeout, tornando-o claro para todos os membros da equipe. Sugere-se realizar treinamentos periodicamente, envolvendo toda a equipe.

No processo de capacitação, os profissionais da saúde muitas vezes não são preparados para avaliar e prevenir erros, se tornando essa lacuna uma das maiores barreiras. Desse modo, a educação e a supervisão permanente dos profissionais são fundamentais, com a finalidade de proporcionar o crescimento pessoal e profissional dos mesmos. A prevenção de eventos adversos é um pré-requisito para a segurança do paciente. Estudo afirma que a campanha realizada junto aos profissionais de saúde vem acarretando melhorias nos indicadores de qualidade ${ }^{11,18,19}$.

Não existem exceções que descartem a aplicação do timeout. O instrumento deve ser aplicado mesmo antes de cirurgias e procedimentos de emergência. Sobre isso, 25 participantes concordam, enquanto 13 concordam em parte. É compreensível que os profissionais demonstrem receio ao concordar que o timeout pode e deve ser utilizado mesmo em cirurgias de emergência, haja vista a preciosidade do tempo nessas ocasiões. Quatro dos 07 Enfermeiros que participaram do estudo fazem parte da percentagem que concorda em parte com a aplicação do timeout em qualquer procedimento ou situação.

É sabido que, em uma situação de urgência/emergência, a equipe cirúrgica deve agir de forma coordenada e rápida, garantindo um atendimento eficiente. Neste caso, a marcação não é obrigatória e o médico responsável deverá fazer a anotação no prontuário posteriormente. Vivenciar um momento de urgência e emergência exige do profissional uma postura de autocontrole, agilidade e competência para enfrentar situações de pacientes que estão no limiar entre a vida e a morte? ${ }^{7}$ Por isso, são compreensíveis as respostas de alguns profissionais, mas não é justificável a não aplicação do protocolo.

A comunicação é um item importante em qualquer ambiente de trabalho e isso não é diferente no CC. Sobre esta questão, 31 participantes do estudo acham que o timeout facilita a comunicação entre os membros da equipe; 05 consideram que o instrumento facilita em parte a comunicação e 03 acham que o timeout não a facilita. Todo o processo de conferência é realizado verbalmente, em voz alta e com a participação da totalidade dos membros da equipe cirúrgica, sendo requerida a interrupção de toda e qualquer atividade em sala. Caso algum item checado esteja 'não conforme', o procedimento deve ser interrompido até sua regularização. Estudos demonstram que a implantação do protocolo ajuda a prevenir a ocorrência de eventos adversos, 
facilitando a comunicação entre os membros da equipe, e, consequentemente, melhorando a assistência prestada ao paciente $^{11,14}$.

As questões finais do questionário, em consonância com o segundo objetivo desta pesquisa, se relacionavam às facilidades e às dificuldades para aplicação do timeout, segundo a opinião dos 39 integrantes da equipe cirúrgica.

Os três fatores que mais facilitam a aplicação do timeout são: a Colaboração da equipe médica, considerada por 25 profissionais, sendo 09 Médicos, 05 Enfermeiros, 10 Técnicos de Enfermagem e 01 Instrumentador Cirúrgico; o Conhecimento do protocolo, considerado facilitador por 15 profissionais, sendo 06 Médicos, 04 Enfermeiros e 05 Técnicos de Enfermagem; e a Clareza e objetividade do protocolo, considerada por 10 profissionais, sendo 06 Médicos, 01 Enfermeiro, 02 Técnicos de Enfermagem e 01 Instrumentador Cirúrgico.

A colaboração da equipe médica mostra que quando estes profissionais não se opõem no momento da aplicação do protocolo e quando respondem prontamente às perguntas, a tarefa torna-se mais rápida, fácil e eficiente. $\mathrm{O}$ trabalho interdisciplinar, realizado com toda a equipe do CC, é de suma importância, tornando-se um dos passos para o sucesso do processo, podendo, assim, atingir a segurança do paciente e a excelência no atendimento. $\mathrm{O}$ comprometimento e o envolvimento da equipe são fundamentais para a melhoria da assistência, sendo esses profissionais responsáveis por seu próprio conhecimento e atualização ${ }^{11,20}$.

$\mathrm{Na}$ análise dos Quadros 1 e 2, constata-se que os Médicos apontaram mais facilidades do que dificuldades, principalmente pontos positivos relacionados à colaboração da equipe e negativos no sentido do timeout ser longo e repetitivo. Os Instrumentadores destacaram facilidades e dificuldades na mesma frequência. Já os Enfermeiros e os Técnicos apontaram mais dificuldades do que facilidades, com pequena diferença. Entre as facilidades, destacase a colaboração da equipe médica e, em contrapartida, grande número de dificuldades também relativas à falta de colaboração da equipe.

Os três fatores que mais dificultam a aplicação do timeout, na opinião da equipe, são: a Falta de colaboração da equipe médica, considerada por 19 profissionais, sendo 02 Médicos, 04 Enfermeiros, 12 Técnicos de Enfermagem e 01 Instrumentador Cirúrgico; o Timeout longo e repetitivo, considerado dificultador por 13 profissionais, sendo 07 Médicos, 02 Enfermeiros e 04 Técnicos de Enfermagem; e os Atrasos, tanto da equipe médica quanto das cirurgias, considerado por 09 profissionais, sendo 05 Médicos, 02 Enfermeiros e 02 Técnicos de Enfermagem.

De acordo com os Técnicos de Enfermagem e os Enfermeiros que fizeram parte da pesquisa, a não colaboração da equipe médica representa a maior dificuldade no momento da aplicação do timeout.

Percebe-se que a implantação destas políticas pode enfrentar barreiras organizacionais e culturais, especialmente por parte dos próprios profissionais ${ }^{11}$. Em um estudo realizado num grande hospital público de São Paulo, os autores consideram que as maiores barreiras são a falta de treinamento da equipe, a não adesão dos profissionais ao protocolo e o não comprometimento da instituição ${ }^{11}$.

Os médicos acham o timeout longo, repetitivo, inclusive com algumas perguntas desnecessárias, e isso é um fator que dificulta e até desmotiva sua aplicação. Porém, a proposta das organizações internacionais ${ }^{1,3-6}$ é que justamente seja realizado um checklist institucional 'universal', que possa ser aplicado a todos e em todas as situações, o que pode gerar repetitividade.

As críticas levantadas pelos integrantes da equipe cirúrgica a respeito do timeout foram: checklist longo e repetitivo; protocolo padronizado para todos os tipos e portes de cirurgias; algumas perguntas que assustam o paciente cirúrgico, como perda esperada de sangue e possibilidade de via aérea difícil; timeout realizado em cirurgias de emergência e após início do procedimento.

As sugestões propostas pela equipe foram: aguardar a montagem completa da sala cirúrgica antes de colocar o paciente em sala; pedir para que o Enfermeiro permaneça na sala operatória no início do procedimento; minimizar atrasos na internação e na chegada do paciente ao CC, além de promover melhor conscientização de todos e mais seriedade na hora da aplicação do timeout.

Mostra-se interessante a observação de alguns profissionais de que certas perguntas assustam os pacientes, a exemplo da possibilidade de sangramento e da existência de via aérea difícil.

\section{Conclusões}

A análise das respostas dos 39 integrantes da equipe cirúrgica (14 Médicos, 14 Técnicos em Enfermagem, 07 Enfermeiros e 04 Instrumentadores Cirúrgicos) demonstrou que:

- Todos os participantes conhecem o timeout (39 ou $100,0 \%)$; os profissionais reconhecem sua eficácia (36 ou 92,3\%); acreditam que sua aplicação traz segurança para o cliente $(37$ ou 94,9), traz segurança para a própria equipe (35 ou 89,7\%); sempre aplicam o protocolo (33 ou $84,6 \%$ ); acham o timeout simples e de fácil compreensão (27 ou 69,2\%); concordam que é passível de ser utilizado em todos os procedimentos e diante de quaisquer situações ( 25 ou $64,1 \%$ ), e, ainda, que facilita a comunicação entre a equipe (31 ou $79,5 \%)$;

- Quanto aos fatores que facilitam a aplicação do timeout, os de maior incidência foram: colaboração da equipe médica ( 25 ou $64,1 \%$ ), conhecimento do protocolo (15 ou 38,5\%) e clareza e objetividade do protocolo (10 ou 25,6\%);

- Entre os fatores que dificultam a aplicação do timeout, destacaram-se: falta de colaboração da equipe médica (19 ou $48,7 \%$ ), protocolo longo e repetitivo (13 ou 33,3\%) e atrasos dos médicos e das cirurgias (09 ou 23,1\%). 


\section{Referências}

1. Organização Pan-Americana da Saúde - OPAS. Aliança mundial para a segurança do paciente - Segundo desafio global para a segurança do paciente. Cirurgias seguras salvam vidas [internet]. Brasília: OPAS; 2009 [citado 2011 Jun 7]. Disponível em: http:// pesquisa.proqualis.net/resources/000000483.

2. Bork AM. Metas internacionais sobre segurança do paciente. Nursing [Internet]. 2007 [citado 2012 Jul 20]. Disponível em: http://www.nursing.com.br/article.php?a=46

3. World Health Organization - WHO. World Health Assembly Resolution and related documents - Quality of care: patient safety (WHA55.18). 18 May 2002 [cited 2012 Apr 24]. p. 2. Available from: http://apps.who.int/gb/archive/pdf_files/WHA55/ewha5518. pdf.

4. World Health Organization - WHO. Patient safety [Internet]. WHO; 2012 [cited 2011 Oct 20]. Available from: http://www. who.int/patientsafety/safesurgery/.

5. Joint Commission International - JCI. Facts about the universal protocol [Internet]. Washington; 2003 [citad $2011 \mathrm{Jul} 20$ ]. Available from: http://www.jointcommission.org/assets/1/18/ universal\%20protocol\%201\%204\%2011PDF

6. Joint Commission on Accreditation of Healthcare Organizations - JCAHO. Joint Commission Internactional. Padrões de acreditações da Joint Commission International para hospitais [Internet]. 4. ed. Rio de Janeiro: Consórcio Brasileiro de Acreditação; 2010 [citado 2011 Ago 25]. Disponível em: www. jointcommissioninternational.org/.../Hospital/Fourth_Edition_ Hospital_Manual_Portuguese_Translation.pdf.

7. Sociedade Beneficente Israelita Brasileira Albert Einstein - SBIBAE. Identificação de sítio cirúrgico (lateralidade) e time out: prevenção de cirurgia e procedimentos invasivos em local de intervenção errado, procedimento errado ou paciente errado [Internet]. 2009 Mar [citado 2012 Mar 12]. Disponível em: http:// medicalsuite.einstein.br/diretrizes_perioperatorios_timeout.pdf.

8. Sociedade Beneficente Israelita Brasileira Albert Einstein - SBIBAE. Segurança em cirurgias e procedimentos invasivos - Identificação de sítio cirúrgico (lateralidade), checklist cirúrgico e time out [Internet]. 2012 Jan [citado 2012 Mar 12]. Disponível em: http:// notsv0/ISO9000/PO.NSF/JCWT/C9CEC B1F9FFC837803256?OpenDocument.

9. Conselho Regional de Enfermagem do Estado de São Paulo - COREN-SP. Rede Brasileira de Enfermagem e Segurança do Paciente - Polo São Paulo - REBRAEN-SP. 10 Passos para a Segurança do Paciente. São Paulo: COREN; 2010. Passo 4, Cirurgia segura. p. 1-3.
10. Gomes AQF. Iniciativas para segurança do paciente difundidas pela Internet por organizações internacionais: estudo exploratório [dissertação]. Rio de Janeiro: Fundação Osvaldo Cruz; 2008 [citado 2011 Ago 10]. Disponível em: http://bases.bireme.br/ cgi-bin/wxislind.exe/iah/online/?IsisScript=iah/iah.xis\&src=goo

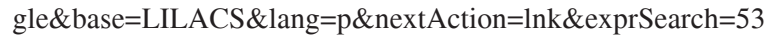
5884\&indexSearch=ID.

11. Vendramini RCR, Silva EA, Ferreira KASL, Possari JF, Baia WRM. Segurança do paciente em cirurgia oncológica: experiência do Instituto do Câncer do Estado de São Paulo. Rev Esc Enferm USP [Internet] 2010 Set [citado 2011 Jun 20];44(3):827-32. Disponível em: http://www.scielo.br/scielo.php?script=sci_artte xt\&pid=S0080-62342010000300039.

12. Ferraz M. A cirurgia segura: uma exigência do Século XXI. Rev Col Bras Cir 2009;36(4):281-2. PMid:20076914. http://dx.doi. org/10.1590/S0100-69912009000400001

13. Paiva MCMS, Paiva SAR, Berti HW. Eventos adversos: análise de um instrumento de notificação utilizado no gerenciamento de enfermagem. Rev Esc Enferm USP. 2010;44:287-94. http://dx.doi. org/10.1590/S0080-62342010000200007

14. Salman FC. A importância da realização do time-out para a segurança no período perioperatório. Anestesia Segura, Serviços Médicos de Anestesia, São Paulo. Disponível em: http://www. anestesiasegura.com/2011/01/importancia-da-realizacao-dotime-out.html.

15. Pereira BMT, Pereira AMT, Correia CS, Marttos AC Jr, Fiorelli RKA, Fraga GP. Interrupções e distrações na sala de cirurgia e trauma: entendendo a ameaça do erro humano. Rev Col Bras Cir. 2011 set-out;38(5):292-8. PMid:22124638. http://dx.doi. org/10.1590/S0100-69912011000500002

16. Landrigan CP. Condições de trabalho e bem-estar dos profissionais de saúde: compartilhamento de lições internacionais para melhorar a segurança do paciente. J Pediatr. 2011 nov-dez;87(6):463-5.

17. Souza LP, Bezerra ALQ, Silva AEBC, Carneiro FS, Paranaguá TTB, Lemos LF. Eventos adversos: instrumento de avaliação do desempenho em centro cirúrgico de um hospital universitário. Rev. Enferm. UERJ. 2011 jan-mar;19(1):127-33. Disponível em: http:// www.facenf.uerj.br/v19n1/v19n1a21.pdf.

18. Badessa GG. Anestesia $4^{\circ}$ passo: time out (check-list de cirurgia segura). Anestesia Segura, Serviços Médicos de Anestesia, São Paulo. Disponível em: http://www.anestesiasegura. com/2010_06_01_archive.html.

19. Harada MJCS, Pedreira MLG, Peterlini MAS, Pereira SR, organizadores. O erro humano e a segurança do paciente. São Paulo: Atheneu; 2006.

20. Lima LF, Leventhal LC, Fernandes MPP. Identificando os riscos do paciente hospitalizado. Einstein. 2008;6(4):434-8. 